\title{
Molecular Mechanism of Tumorigenesis and Biomarkers of Opisthorchiasis-associated Cholangiocarcinoma
}

\section{Zhiliang $\mathrm{Wu}^{1 *}$ and Thidarut Boonmars ${ }^{2}$}

${ }^{1}$ Department of Parasitology, Gifu University Graduate School of Medicine, Japan

${ }^{2}$ Liver Fluke and Cholangiocarcinoma Research Center, Faculty of Medicine, Khon Kaen University, Thailand

\begin{abstract}
Definitive relationship has been confirmed between Opisthorchis viverrini infection and tumorigenesis of cholangiocarcinoma (CCA), by epidemiological investigation and animal experiments. In the O. viverrini endemic area, opisthorchiasis-associated CCA has been becoming a serious public health problem. Understanding of the molecular mechanisms of tumorigenesis of the infection-induced CCA will be helpful to develop new biomarkers for diagnosis and therapy. This review addresses recent progresses in molecular mechanism and biomarker of opisthorchiasisassociated CCA.
\end{abstract}

Keywords: Tumorigenesis; $\quad$ Opisthorchiasis-associated cholangiocarcinoma

\section{Introduction}

O. viverrini is a human liver fluke and mainly endemic in the Lower Mekong Basin, including Thailand, Lao PDR, Vietnam and Cambodia. The correlation of the high prevalence of $O$. viverrini infection with the high incidence of cholangiocarcinoma (CCA) in endemic areas has suggested the definitive relationship between them. Serious O. viverrini infection in endemic area plus the lack of CCA early diagnostic techniques and poor prognosis put the millions of people at the risk of $\mathrm{CCA}$. Moreover, as the second most common primary liver cancer in the non-endemic area of $O$. viverrini, the incidence and mortality of CCA are markedly increasing in recent decades. An unexpected scenario was reported in 2012 that the mortality of CCA of the workers in one printing company in Japan was about 2900 times higher compared with that in the normal population.

CCA is a quite lethal cancer, with a poor prognosis and limitation in therapeutic options. It is usually difficult to make an early diagnosis for CCA, because in its early stages, there are no specific symptoms or physical signs, and abnormal in laboratory examination, as well as tumor markers with high sensitivity and specificity. Moreover, special diagnostic strategy is necessary for the opisthorchiasis-associated CCA because this type of CCA is usually intrahepatic, characterized with the neoplasm in upper hepatoduodenal ligament, and extension into liver and vascular structure. Inability to diagnose CCA early also makes it difficult for therapeutic options, as patients are usually in their advanced stages, with the existence of distant and extensive metastasis when diagnosed. Therefore, it is urgent to delve into the tumorigenesis mechanism and develop novel biomarkers for the diagnosis, prognosis, metastasis and therapy of the CCA. The present study emphasizes on the recent progresses of molecular mechanism of tumorigenesis and biomarkers in opisthorchiasis-associated CCA.

\section{Current Status of $O$. viverrini Prevalence}

Adult worm of O. viverrini inhabits in the biliary system of final hosts, including human and reservoir (cats and dogs in endemic regions). Individuals are infected by eating raw or uncooked fish, which is intermediate host infected by themetacercariae of $O$. viverrini. The high prevalence of $O$. viverrini infection is associated with the exposure to the infected fish, accustom and culture in the local endemic areas. High infective rate of the fish was reported in the endemic areas, for example, $12.3 \%$ in Nakhon Ratchasima and $46.9 \%$ in Khon Kaen Province of Thailand [1,2], 14.5\% in Lao PDR [3]. The heavy prevalence is considered to be continued from past time, as the first investigation reported a $100 \%$ infection of O. viverrini in some region of northeast Thailand [4], and even in 1980's, the prevalence was still near $100 \%$ in Khon Kaen Province in northeast Thailand, whereas now still the region with the highest prevalence in the world [5-7]. In recent decades, several control programs have been implemented to control the infection. Although improved, the prevalence is still in its high situation. In northeast Thailand, prevalence was $34.6,15.7$ and $16.6 \%$ in 1998 , 2000 and 2009, respectively, and in some region, the prevalence was as high as $60 \%$ [8]. It is estimated there are about 6 million individuals to be infected with $O$. viverrini, but the numbers is considered to be underestimated due to the diagnostic sensitivity.

High prevalence of O. viverrini was also reported in Lao PDR. A nationwide survey in 2003 showed an average infection of 10.9\% in schoolchildren, and in some region of the Mekong River Basin, the prevalence was more than $60-80 \%$ [9]. A more recent investigation showed the prevalence as high as $90.9-92 \%$ in the lowland areas [10]. It was estimated that there are 2.5 million individuals to be infected, accounting for $37 \%$ of the population. Little is known on the prevalence of O. viverrini in Cambodian, but recent investigation showed 32.0$44.8 \%$ prevalence in Takeo Province [11]. The infection rate of cyprinid fish was as high as $66.7 \%$ in some areas [12]. Similarly, in Vietnam, the prevalence has been found to range from $15.2 \%$ to $36.9 \%$ in some endemic areas [13].

\section{Opisthorchiasis-associated CCA}

The habitat of O. viverrini is in hepatobiliary tract, bile duct and gallbladder, and opisthorchiasis is caused from mechanical irritation of the fluke and immunopathogenesis induced by theirexcretory/secretory

*Corresponding author: Zhiliang Wu, Department of Parasitology, Gifu University Graduate School of Medicine, Japan, E-mail: wu@gifu-u.ac.jp

Received March 04, 2013; Accepted May 28, 2013; Published June 07, 2013

Citation: Wu Z, Boonmars T (2013) Molecular Mechanism of Tumorigenesis and Biomarkers of Opisthorchiasis-associated Cholangiocarcinoma. J Bacterio Parasitol 4: 165. doi:10.4172/2155-9597.1000165

Copyright: (c) 2013 Wu Z, et la. This is an open-access article distributed unde the terms of the Creative Commons Attribution License, which permits unrestricted use, distribution, and reproduction in any medium, provided the original author and source are credited. 
(ES) products that are immunogenic, mitogenic and toxic to the biliary epithelium, which leads to chronic inflammation, desquamation, epithelial and adenomatous hyperplasia, goblet cell metaplasia, periductal fibrosis and granuloma formation [14-16]. About 25\% of O. viverrini infected cases develops into priductal fibrosis and some of them develops into CCA.

Epidemiological investigation indicated that the CCA morbidity is positively correlated to O. viverrini prevalence $[17,18]$. The highest morbidity of CCA in the world was found in the northeast of Thailand, where is heavy endemic area of $O$. viverrini infection with the highest prevalence in the world [18-21]. The evidence from animal experiment has also confirmed that $O$. viverrini infection could induce occurrence of CCA. Infection with $O$. viverrini only, or administration with NDMA at low concentration in limited time does not induce CCA development, but infection plus NDMA administration does induce CCA development [22]. Now, this is the only animal model of O. viverrini infection-induced CCA, and is applied widely. Therefore, O. viverrini has been recognized as group 1 carcinogen by the International Agency for Research on Cancer (IARC) of the WHO in 1994, which means the parasitic infection is considered to be a direct risk factor for CCA.

Although CCA is a rear cancer in non-endemic areas, the incidence of $O$. viverrini in endemic areas is extremely higher than that in nonendemic regions [18]. Estimated, in northeast Thailand, 5000 cases of CCA is diagnosed annually [21]. High mortality of liver cancer was observed in northeast Thailand, where nine out of the ten provinces ranked in the top death numbers of liver cancer in Thailand [23].

\section{Molecular Mechanism of Tumorigenesis Opisthorchiasis-associated CCA}

The mechanisms of CCA tumorigenesis induced by O. viverrini infection remain vague. It is generally considered that mechanical injury and parasite-derived products are initial causes. The excretory-secretory (ES) products from the adults are immunogenic, mitogenic and toxic. Inflammation induced by these products is linked with hepatobiliary pathology. NOS derived from inflammation by the infection are one of factors to cause the tumorigenesis. It has been demonstrated that $O$. viverrini caused the accumulation of NOS [24]. The accumulated NOS may induce the DNA damage, such as epigenetic changes and genomic instability, which may initiate the tumorigenesis of CCA. The oxidative DNA damage has been observed during $O$. viverrini infection. Infection caused the increase of expression of 8-nitroguanine and 8-oxo-7,8dihydro-20-deoxyguanosine in the inflammatory cells and epithelial lining of bile duct at inflammatory areas in the liver, which is considered to mediate iNOS dependent DNA damage in intrahepatic bile duct epithelium [24-26]. cDNA microarray analysis indicated that in the animal model of $O$. viverrini infection-induced CCA, the expressions of many genes related to antioxidative enzyme were down-regulated, for example, Idh, Hgd, Gsta, Gss, Prdx6 and Cyp [27]. Some studies have found that the ES products of $O$. viverrini have direct effect on cell proliferation [28], by up-regulating the expression of proliferation factors, such as Pdgf, Tgf and EGF [29].

Great progresses have been made in determining the molecular mechanism of the tumorigenesis of opisthorchiasis-associated CCA, by using animal model. Utilizing the CCA model, a systemic cDNA microarray analysis listed up the candidate genes that may be involved in tumorigenesis, including the genes related to cell proliferation, differentiation and transformation, cell growth and cycle regulation, apoptosis, DNA repair, cytoskeletal structure, metabolic enzymes, tumor suppressor and oxidative reduction response [27]. Several signal pathways were identified to be likely involved in the tumorigenesis.

Proliferation, differentiation and transformation are the key characterizations in tumorigenesis process. Study in O. viverrini infection-induced CCA in animal model revealed the genes related to cell proliferation, differentiation and transformation may play role in the tumorigenesis of the CCA, for example, S100a6, Anxa2, Pdgfa, Frat1, Lifr, Npdc1, Enc1, Cgref1, Tgfb2, Klf4, Maff, Jund1 and Cebpd [27]. Correspondingly, some of O. viverrini-derived factors have been identified to promote the proliferation of host cell, for example, granulin-like growth factor [30] and glutathione S-transferase [31]

NFkB signaling pathway is one of the important pathways to involve in inflammation-induced carcinogenesis. There were up-regulated expressions of NFkB, NFkBIa, NFkBIb, and the cycle regulatory gene cyclin D1, cyclin E, and CDK4 during tumorigenesis of O. viverrini infection-induced CCA in animal model [27]. Moreover, ES from $O$. viverrini induced $\mathrm{IkB}$ degradation and activated NF-kB unclear translocation [32].

$\mathrm{RB}$ pathway plays important role in controlling G1/S transition in the cell cycle. This pathway includes the four key genes of RB1, p16 $16^{\mathrm{INK}}$, cyclin D1 and CDK4. Inactivation of RB pathway was found in various kinds of human cancers. Alternation of the expression of the genes was observed in O. viverrini infection-induced CCA animal model, and human CCA. The expressions of RB1 and $\mathrm{p} 16^{\mathrm{INK} 4}$ were down-regulated, and the expressions of cyclin D1 and CDK4 were up-regulated during the development of CCA [33].

c-Ski is a repressor of TGF- $\beta$ signal pathway. As an oncogene, c-Ski was characterized by its ability in cell differentiation and transformation. C-Ski protein levels and subcellular localization were found to correlate with clinicopathological parameters and tumor progression in several human malignancies. In the tumorigenesis of $O$. viverrini infectioninduced CCA in animal model, the kinetic expression of c-ski and TGF- $\beta$ signal pathway genes was corresponding to the time-dependent development of the CCA, suggesting that c-ski is likely involved the tumorigenesis as a repressor of TGF- $\beta$ signaling pathway, through inhibiting the pathway-induced growth barrier [34].

More recently, it was reported that PDGF signaling pathway may play important roles in opisthorchiasis-associated CCA tumorigenesis [35,36]. PDGF/PDGFR complex engages several well known signaling pathways, and plays the pivotal roles in cell proliferation, differentiation, transformation, invasion and survival. In O. viverrini infection-induced CCA animal model, the expression of Pdgfa and its receptor Pdgfra was found to be up-regulated during tumorigenesis [27,35]. Further analysis indicated that these factors were up-regulated in human CCA tissue $(84.6 \%)$, collected from heavy endemic area of O. viverrini. Positive PDGFA immunohistochemical staining was significantly correlated with status, stage and survival rate of CCA. Moreover, the serum level of PDGFA in CCA patients was significantly higher than that in healthy control, suggesting its potential as prognostic and diagnostic markers [36]. These studies indicated that PDGF signaling pathway likely involves in tumor angiogenesis, maintenance of the tumor microenvironment, and implication in the development and metastasis of the CCA, as in other tumors [37-39].

Galectin-1 functions in cell adhesion, proliferation, differentiation, and has been demonstrated to be involved in tumor progression and metastasis. Study has indicated that the expression of galectin-1, at both mRNA and protein levels, was greatly elevated with the tumor 
progression of opisthorchiasis-associated CCA in animal model and human CCA. The location of the increased expression further suggested the involvement of galectin-1 in the CCA tumorigenesis, as the increased expression of the protein was mainly located in the epithelium of extensively proliferated and hyperplasia small bile ducts at early stage of CCA development, and in the extensive tumor stroma tissues at later stage, in both model animals and human CCA cases [40].

\section{Biomarkers of Opisthorchiasis-associated CCA}

Conventional serum markers, such as Carcinoembryonic antigen (CEA) and carbohydrate antigen 19-9 (CA19-9), are now used in the diagnosis of malignancies including in CCA. However, these markers are limited in diagnosis of CCA due to their insufficient sensitivity and specificity. Recent years, the progress has been made on the development of biomarkers of opisthorchiasis-associated CCA. Sripa et al. [41] reported that an elevation of plasma level of IL-6 in patients is associated with a significant increase in the risk of opisthorchiasisassociated CCA and advanced periductal fibrosis, which is also linked to high risk of CCA. O. viverrini infection alone did not cause the increase of IL-6 levels, as revealed by low level IL-6 in O. viverrini infected individuals without advanced periductal fibrosis or CCA, suggesting IL- 6 may be a novel biomarker for diagnosis and monitoring the response to therapies in the lethal pathology induced by O. viverrini infection.

Variability of animal model of O. viverrini infection-induced CCA enhances the development of revealing tumorigenesis mechanism and exploring novel biomarkers for CCA. Several potential biomarkers have been identified in the animal model. PDGFA and PDGFRA were reported to be elevated in the animal model and the tumor tissue of CCA cases [36]. Cox repression multivariate analysis indicated that positive immunostaining of PDGFA had a higher likelihood of risk of death, and the serum level in CCA patients was higher than in healthy individuals, suggesting its potential as a prognostic and diagnostic marker. The anti-cancer drug sunitibib malate inhibited the proliferation of CCA cell by suppressing PDGFRA signaling pathway, suggesting its potential as a target of therapy. Similarly, the increased expression galectin-1 was observed during tumorigenesis of $O$. viverrini infection-induced CCA, and in the tumor stroma of CCA cases [40]. High positive rate of immunostaining and correlation with stage, metastasis and cumulative overall survival indicated that galectin- 1 is likely involved in the tumorigenesis, and expected to serve as a tumor stroma marker in diagnosis and prediction of metastasis and poor prognosis of the opisthorchiasis-associated CCA.

Many other biomarkers were also found to be promising as candidate markers of opisthorchiasis-associated CCA recently, for example hydroxyproline as a diagnostic marker [42], ECPKA and its autoantibody as biomarkers [43], oxysterols (OSBP2 and OSBPL-7) as molecular markers for the identification of CCA metastasis in the bloodstream [44], and ANXA2 as prognostic marker [45].

Silsirivanit et al. [46] and Sawanyawisuth et al. [47] reported an novel CCA antigens, an unidentified glycan epitope on MUC5AC and designated as CCA-associated carbohydrate antigen (CCA-CA), which was found to be up-regulated at early stage in CCA development, suggesting the possibility of this antigen as an early diagnostic and prognostic marker of CCA.

\section{Perspective}

Great progresses have been made in revealing molecular mechanisms of tumorigenesis and developing biomarkers for diagnosis, prognosis and therapy, particularly in recent years; but they are still primitive. By utilizing new developed techniques in the research of opisthorchiasis-associated CCA, it will accelerate the progress to identify new biomarkers. More important, it is unlike other type cancers, opisthorchiasis-associated CCA has its animal model which does closely mimic human CCA, which provides a desirable platform to deeply explore the mechanisms of opisthorchiasis-associated CCA. Taken together, it is believed that demystification of tumorigenesis of opisthorchiasis-associated CCA and development of applicable biomarkers for the early diagnosis and therapy will be promising in the future.

\section{Acknowledgments}

This research was supported by the Grant-in-Aid for Scientific Research (24590504), from the Ministry of Education, Culture, Sports, Science and Technology of Japan. The authors are thankful to Dr. Jianxin Sun, an epidemiologist at Connecticut Department of Public Health, for his critical reading and editing of this manuscript.

\section{References}

1. Kaewpitoon N, Kaewpitoon SJ, Ueng-arporn N, Rujirakul R, Churproong S et al. (2012) Carcinogenic human liver fluke: current status of Opisthorchis viverrini metacercariae in Nakhon Ratchasima, Thailand. Asian Pac J Cancer Prev 13: 1235-1240

2. Pitaksakulrat O, Sithithaworn P, Laoprom N, Laha T, Petney TN, et al. (2013) A cross-sectional study on the potential transmission of the carcinogenic liver fluke Opisthorchis viverrini and other fishborne zoonotic trematodes by aquaculture fish. Foodborne Pathog Dis 10: 35-41.

3. Rim HJ, Sohn WM, Yong TS, Eom KS, Chai JY, et al. (2013) Fishborne trematode metacercariae in Luang Prabang, Khammouane, and Saravane Province, Lao PDR. Korean J Parasitol 51: 107-114.

4. SADUN EH (1955) Studies on Opisthorchis viverrini in Thailand. Am J Hyg 62 81-115.

5. Upatham ES, Viyanant V, Kurathong S, Rojborwonwitaya J, Brockelman WY, et al. (1984) Relationship between prevalence and intensity of Opisthorchis viverrini infection, and clinical symptoms and signs in a rural community in north-east Thailand. Bull World Health Organ 62: 451-461.

6. Upatham ES, Viyanant V, Kurathong S, Brockelman WY, Menaruchi A, et al (1982) Morbidity in relation to intensity of infection in Opisthorchiasis viverrini: Study of a community in KhonKaen, Thailand. Am J Trop Med Hyg 31: 1156 1163.

7. Upatham ES, Brockelman WY, Viyanant V, Lee P, Kaengraeng R, et al. (1985) Incidence of endemic Opisthorchis viverrini infection in a village in northeast Thailand. Am J Trop Med Hyg 34: 903-906.

8. Sithithaworn P, Andrews RH, Nguyen VD, Wongsaroj T, Sinuon M, et al. (2012) The current status of opisthorchiasis and clonorchiasis in the Mekong Basin Parasitol Int 61: 10-16.

9. Rim HJ, Chai JY, Min DY, Cho SY, Eom KS, et al. (2003) Prevalence of intestinal parasite infections on a national scale among primary schoolchildren in Laos. Parasitol Res 91: 267-272.

10. Sayasone S, Mak TK, Vanmany M, Rasphone O, Vounatsou P, et al. (2011) Helminth and intestinal protozoa infections, multiparasitism and risk factors in Champasack province, Lao People's Democratic Republic. PLoS Negl Trop Dis 5: e1037.

11. Sohn WM, Kim HJ, Yong TS, Eom KS, Jeong HG, et al. (2011) Echinostoma ilocanum infection in Oddar Meanchey Province, Cambodia. Korean J Parasito 49: $187-190$

12. Touch S, Komalamisra C, Radomyos P, Waikagul J (2009) Discovery of Opisthorchis viverrini metacercariae in freshwater fish in Southern Cambodia. Acta Trop 111: 108-113.

13. De NV, Murrell KD, Cong le D, Cam PD, Chau le V, et al. (2003) The food-borne trematode zoonoses of Vietnam. Southeast Asian J Trop Med Public Health 34: 12-34.

14. Sripa B (2003) Pathobiology of opisthorchiasis: an update. Acta Trop 88: 209 220. 
Citation: Wu Z, Boonmars T (2013) Molecular Mechanism of Tumorigenesis and Biomarkers of Opisthorchiasis-associated Cholangiocarcinoma. J Bacteriol Parasitol 4: 165. doi:10.4172/2155-9597.1000165

15. Sripa B, Kaewkes S, Sithithaworn P, Mairiang E, Laha T, et al. (2007) Liver fluke induces cholangiocarcinoma. PLoS Med 4: e201.

16. (1994) Infection with liver flukes (Opisthorchis viverrini, Opisthorchis felineus and Clonorchis sinensis). IARC Monogr Eval Carcinog Risks Hum 61: 121-175.

17. Shin HR, Oh JK, Masuyer E, Curado MP, Bouvard V, et al. (2010) Epidemiology of cholangiocarcinoma: an update focusing on risk factors. Cancer Sci 101 579-585.

18. Sripa B, Brindley PJ, Mulvenna J, Laha T, Smout MJ, et al. (2012) The tumorigenic liver fluke Opisthorchis viverrini--multiple pathways to cancer. Trends Parasitol 28: 395-407.

19. Srivatanakul P, Ohshima H, Khlat M, Parkin M, Sukaryodhin S, et al. (1991) Opisthorchis viverrini infestation and endogenous nitrosamines as risk factors for cholangiocarcinoma in Thailand. Int J Cancer 48: 821-825.

20. Vatanasapt V, Uttaravichien T, Mairiang EO, Pairojkul C, Chartbanchachai W, et al. (1990) Cholangiocarcinoma in north-east Thailand. Lancet 335: 116-117.

21. Sripa B, Bethony JM, Sithithaworn P, Kaewkes S, Mairiang E, et al. (2011) Opisthorchiasis and Opisthorchis-associated cholangiocarcinoma in Thailand and Laos. Acta Trop 120: S158-S168.

22. Thamavit W, Pairojkul C, Tiwawech D, Itoh M, Shirai T, et al. (1993) Promotion of cholangiocarcinogenesis in the hamster liver by bile duct ligation after dimethylnitrosamine initiation. Carcinogenesis 14: 2415-2417.

23. Sripa B, Pairojkul C (2008) Cholangiocarcinoma: lessons from Thailand. Curr Opin Gastroenterol 24: 349-356.

24. Pinlaor S, Yongvanit P, Hiraku Y, Ma N, Semba R, et al. (2003) 8-nitroguanine formation in the liver of hamsters infected with Opisthorchis viverrini. Biochem Biophys Res Commun 309: 567-571.

25. Pinlaor S, Hiraku Y, Ma N, Yongvanit P, Semba R, et al. (2004) Mechanism of NO-mediated oxidative and nitrative DNA damage in hamsters infected with Opisthorchis viverrini: A model of inflammation-mediated carcinogenesis. Nitric Oxide 11: 175-183.

26. Kawanishi S, Hiraku Y, Pinlaor S, Ma N (2006) Oxidative and nitrative DNA damage in animals and patients with inflammatory diseases in relation to inflammation-related carcinogenesis. Biol Chem 387: 365-372.

27. Wu Z, Boonmars T, Boonjaraspinyo S, Nagano I, Pinlaor S, et al. (2011) Candidate genes involving in tumorigenesis of cholangiocarcinoma induced by Opisthorchis viverrini infection. Parasitol Res 109: 657-673.

28. Thuwajit C, Thuwajit P, Kaewkes S, Sripa B, Uchida K, et al. (2004) Increased cell proliferation of mouse fibroblast NIH-3T3 in vitro induced by excretory/ secretory product(s) from Opisthorchis viverrini. Parasitology 129: 455-464.

29. Thuwajit C, Thuwajit P, Uchida K, Daorueang D, Kaewkes S, et al. (2006) Gene expression profiling defined pathways correlated with fibroblast cell proliferation induced by Opisthorchis viverrini excretory/secretory product. World J Gastroenterol 12: 3585-3592.

30. Smout MJ, Laha T, Mulvenna J, Sripa B, Suttiprapa S, et al. (2009) A granulinlike growth factor secreted by the carcinogenic liver fluke, Opisthorchis viverrini, promotes proliferation of host cells. PLoS Pathog 5: e1000611.

31. Daorueang D, Thuwajit P, Roitrakul S, Laha T, Kaewkes S, et al. (2012) Secreted Opisthorchis viverrini glutathione S-transferase regulates cell proliferation through AKT and ERK pathways in cholangiocarcinoma. Parasitol Int 61: 155-161.

32. Ninlawan K, O'Hara SP, Splinter PL, Yongvanit P, Kaewkes S, et al. (2010) Opisthorchis viverrini excretory/secretory products induce toll-like receptor 4 upregulation and production of interleukin 6 and 8 in cholangiocyte. Parasitol Int 59: 616-621.
33. Boonmars T, Wu Z, Boonjaruspinyo S, Pinlaor S, Nagano I, et al. (2009) Alterations of gene expression of RB pathway in Opisthorchis viverrini infectioninduced cholangiocarcinoma. Parasitol Res 105: 1273-1281.

34. Boonmars T, Wu Z, Boonjaruspinyo S, Puapairoj A, Kaewsamut B, et al. (2011) Involvement of $\mathrm{C}$-Ski oncoprotein in carcinogenesis of cholangiocacinoma induced by Opisthorchis viverrini and $\mathrm{N}$-nitrosodimethylamine. Pathol Oncol Res 17: 219-227.

35. Boonjaraspinyo S, Wu Z, Boonmars T, Kaewkes S, Loilome W, et al. (2012) Overexpression of PDGFA and its receptor during carcinogenesis of Opisthorchis viverrini-associated cholangiocarcinoma. Parasitol Int 61: 145150.

36. Boonjaraspinyo S, Boonmars T, Wu Z, Loilome W, Sithithaworn P, et al. (2012) Platelet-derived growth factor may be a potential diagnostic and prognostic marker for cholangiocarcinoma. Tumour Biol 33: 1785-1802.

37. Kartha RV, Sundram UN (2008) Silent mutations in KIT and PDGFRA and coexpression of receptors with SCF and PDGFA in Merkel cell carcinoma: implications for tyrosine kinase-based tumorigenesis. Mod Pathol 21: 96-104.

38. Lagonigro MS, Tamborini E, Negri T, Staurengo S, Dagrada GP, et al. (2006) PDGFRalpha, PDGFRbeta and KIT expression/activation in conventional chondrosarcoma. J Pathol 208: 615-623.

39. Tamborini E, Miselli F, Negri T, Lagonigro MS, Staurengo S, et al. (2006) Molecular and biochemical analyses of platelet-derived growth factor receptor (PDGFR) B, PDGFRA, and KIT receptors in chordomas. Clin Cancer Res 12 6920-6928.

40. Wu Z, Boonmars T, Nagano I, Boonjaraspinyo S, Pinlaor S, et al. (2012) Alteration of galectin-1 during tumorigenesis of Opisthorchis viverrini infectioninduced cholangiocarcinoma and its correlation with clinicopathology. Tumour Biol 33: 1169-1178.

41. Sripa B, Thinkhamrop B, Mairiang E, Laha T, Kaewkes S, et al. (2012) Elevated plasma IL-6 associates with increased risk of advanced fibrosis and cholangiocarcinoma in individuals infected by Opisthorchis viverrini. PLoS Negl Trop Dis 6: e1654

42. Prakobwong S, Charoensuk L, Hiraku Y, Pinlaor P, Pairojkul C et al (2012) Plasma hydroxyproline, MMP-7 and collagen I as novel predictive risk markers of hepatobiliary disease-associated cholangiocarcinoma. Int J Cancer 131 E416-E424.

43. Loilome W, Yooyuen S, Namwat N, Sithithaworn P, Puapairoj A, et al. (2012) PRKAR1A overexpression is associated with increased ECPKA autoantibody in liver fluke-associated cholangiocarcinoma: application for assessment of the risk group. Tumour Biol 33: 2289-2298.

44. Loilome W, Wechagama P, Namwat N, Jusakul A, Sripa B, et al. (2012) Expression of oxysterol binding protein isoforms in opisthorchiasis-associated cholangiocarcinoma: a potential molecular marker for tumor metastasis. Parasitol Int 61:136-139.

45. Yonglitthipagon P, Pairojkul C, Chamgramol Y, Mulvenna J, Sripa B (2010) Up-regulation of annexin A2 in cholangiocarcinoma caused by Opisthorchis viverrini and its implication as a prognostic marker. Int J Parasitol 40: 1203 1212

46. Silsirivanit A, Araki N, Wongkham C, Pairojkul C, Narimatsu Y, et al. (2011) A novel serum carbohydrate marker on mucin 5AC: values for diagnostic and prognostic indicators for cholangiocarcinoma. Cancer 117: 3393-3403.

47. Sawanyawisuth K, SilsirivanitA, Kunlabut K, Tantapotinan N, Vaeteewoottacharn $\mathrm{K}$, et al. (2012) A novel carbohydrate antigen expression during development of Opisthorchis viverrini- Associated cholangiocarcinoma in golden hamster: a potential marker for early diagnosis. Parasitol Int 61:151-154. 